\title{
Increasing the flexibility of European type electricity auctions via a novel bid class
}

\author{
Dávid Csercsik \\ Pázmány Péter Catholic University \\ Faculty of Information Technology and Bionics \\ Email: csercsik@itk.ppke.hu
}

\author{
Ádám Sleisz \\ and Péter Márk Sőres \\ Budapest University of Technology and Economics \\ Department of Power Engineering \\ Email: \{sleisz.adam,sores.peter\} @ vet.bme.hu
}

\begin{abstract}
In the framework of EUPHEMIA, Minimum Income Condition (MIC) orders are supply orders consisting of several hourly step bids for different market hours, bound together by the MIC, which formalizes the requirement that the overall income of the MIC order must cover its given costs, usually composed by a fixed and a variable term. In this paper we introduce a so-called flexible orders, which are also based on the the MIC description, but are composed of mutually exclusive different production profiles. We define the computational formulation of this possible new bid type, and demonstrate the properties of the bid acceptance in the case of two simple two-period examples, considering various market clearing prices (MCPs). We argue that this new type of bid increases the flexibility of day-ahead markets in the presence of price-uncertainty.
\end{abstract}

\section{INTRODUCTION}

The ultimate aim of electricity market reforms in the European Union (EU) is to create a unified internal market for electricity, and recently, also to encourage investments in clean energy technologies [1]. Regarding market integration, the problems of border effects identified in the previous decade [2] are still not solved completely, but much progress has been made. One key topic in the market-unification process is the integration of day-ahead power exchanges (DAPXs). The tool called EUPHEMIA [3] has been proposed to perform the clearing of the all-European electricity market.

In addition, as power generating units - bidding on the supply side of these markets - based on various technologies are subject to different technological and economic constraints, their efficient representation in the power exchanges has always been a challenging task. In particular, these difficulties are originating from the non-convex nature of the costs of power generation [4], such as start-up costs, minimal operating levels and load gradient constraints. The inclusion of concepts related to these considerations into the optimization frameworks of DAPXs is a constant struggle of electricity market design.

The most fundamental approach to this problem is the inclusion of block orders [5, 6] with the so-called fill-orkill property, limiting their possible acceptance only to the values of 0 and 1 (potentially also for multiple periods), without the possibility of partial acceptance. The description of EUPHEMIA [3] also includes the concept of linked and exclusive groups of block orders.
Another approach which allows power plants to represent their fixed (like-start up) and variable (like load-dependent fuel) costs is the concept of minimum income conditions (MIC). A MIC order is basically a set of conventional hourly supply orders with an additional constraint, which ensures that the resulting allocation of the bids belonging to the order must cover the predetermined costs. If the MIC condition is not fulfilled, every bid of the respective order is fully rejected. Clearing algorithms and efficient formulations for such bids are discussed in [7-10].

In addition, price uncertainty may be an other issue implying inefficiencies. As discussed in [11], internalisation of startup and other costs based on market expectations in regular (not MIC) bids may distort the energy offer curve. The paper [12] analyzes the role of price-uncertainty in the reduction of efficiency, and compares simple and block bidding to multipart (MIC-like) bidding. The conclusion of this paper is that even under optimal simple and block bidding generators face the risk of ex-post suboptimal solutions, whereas in multi-part bidding these do not occur, which points to efficiency gains of multi-part bidding in the presence of uncertainty in electricity markets. In general it may be said that under price-uncertainty it is especially desirable to give the generating units more options for simple and especially complex (multipart) bidding to, on the one hand, explicitly account for their start-up costs, and on the other hand, to make their bids more robust against risks arising from price uncertainties.

In this paper we aim to combine the approach of MIC bids with the idea of exclusive groups of block orders to introduce a new type of complex order to increase the flexibility of portfolio-bidding type European DAPXs.

\section{COMPUTATIONAL FORMULATION}

To keep the proposed framework as simple as possible, in the first step we only consider conventional step bids (thus no ramp-bids or multistep-bids).

The basic idea of the proposed concept is that generating units may submit so-called flexible orders, which consist of multiple, mutually exclusive bid profiles, potentially resulting in different generating schedule regarding time periods as well.

In the later subsections we will demonstrate the principle on simple 2-period examples. 
In the notations introduced in the following, the lower index corresponds to the period, while the upper index corresponds to the index of the flexible bid in question and to the index of the bid profile.

The variables denoted by $y$ stand for the acceptance indicators of single bids: $y_{p}^{f i, j}$ denotes the acceptance indicator of the bid corresponding to the $p$-th period of bid profile $j$ defined in the $i$-th flexible order ( $f i$ in the upper index relates the indicator to the $i$-th flexible bid, while the following $-j$ suffix in the upper index refers to the $j$-th bid profile). Similarly $q_{p}^{f i, j}$ and $p_{p}^{f i, j}$ denote the quantity and price of the bid in question. As we consider supply bids, we assume $q_{p}^{f i, j}>0$.

\section{A. Cost model}

The cost model of the generating units in question, described in eq. (1) is the same as in the case of MIC bids in [7]:

$$
T C^{f i}=F^{f i}+\sum_{j, p} V_{p}^{f i} q_{p}^{f i, j} y_{p}^{f i, j}
$$

where $F^{f i}$ represents the fixed cost component of flexible order $i$, which arises if any bid of any submitted production profile is at least partially accepted (start-up cost), while $V_{p}^{f i}$ denotes the variable cost of production. The lower index $p$ relates to the consideration that in contrast to the original MIC concept described in [7], in general we allow for timedependence of this constant. The product $q_{p}^{f i, j} y_{p}^{f i, j}$ is equal to the produced amount corresponding to period $p$ of the $j$-th bid profile.

\section{B. Profile-exclusivity inequalities}

Equation (2) formulates the consideration that the bid profiles are exclusive.

$$
\begin{aligned}
& y_{p}^{f i, j} \leq u^{f i, j} \quad \forall(i, j) \\
& \sum_{j} u^{f i, j} \leq 1 \quad \forall i
\end{aligned}
$$

where $T$ is the total number of periods (in our example $T=2$ ), and $u^{f i, j}$ is a binary variable corresponding to the $j$-th bid profile of the flexible order $i$.

\section{Income}

Let us denote the income of the bid corresponding to $y_{p}^{f i, j}$ by $I_{p}^{f i, j}$. Intuitively $I_{p}^{f i, j}$ may be calculated as

$$
I_{p}^{f i, j}=M C P_{p} q_{p}^{f i, j} y_{p}^{f i, j}
$$

where $M C P_{p}$ stands for the market clearing price of energy in period $p$.

Equation (3) holds however a quadratic expression of variables, namely the product of $M C P_{p}$ and $y_{p}^{f i, j}$, which would result in a computationally demanding quadratically constrained problem (MIQCP). To overcome this issue, following the methodology proposed in [8] we formulate the expressions for income as

$$
\begin{aligned}
y_{p}^{f i, j}>0 \rightarrow I_{p}^{f i, j} & =y_{p}^{f i, j} q_{p}^{f i, j} p_{p}^{f i, j} \\
& +q_{p}^{f i, j} M C P_{p}-q_{p}^{f i, j} p_{p}^{f i, j} \\
y_{p}^{f i, j}<1 \quad \rightarrow \quad I_{p}^{f i, j} & =y_{p}^{f i, j} q_{p}^{f i, j} p_{p}^{f i, j}
\end{aligned}
$$

To elucidate the formulas (4) and (5), let us enumerate the following three possibilities:

- If the bid is entirely accepted ( $\left.y_{p}^{f i, j}=1\right), I_{p}^{f i, j}$ equals the product of $q_{p}^{f i, j}$ and $M C P_{p}$ according to (4).

- If the bid is partially accepted $\left(M C P_{p}=p_{p}^{f i, j}\right), I_{p}^{f i, j}$ equals to $y_{p}^{f i, j} q_{p}^{f i, j} p_{p}^{f i, j}$. Both (4) and (5) are active in this case and they result in the same inequality.

- If the bid is entirely rejected $\left(y_{p}^{f i, j}=0\right)$, according to (5) $I_{p}^{f i, j}=0$.

The total income of the bid profile $j$ of the $i$-th flexible order may be defined as

$$
I^{f i, j}=\sum_{p=1}^{T} I_{p}^{f i, j}
$$

\section{Minimum income condition}

According to the previous assumptions, the minimum income condition may be formulated as

$$
u_{p}^{f i, j}>0 \rightarrow I^{f i, j} \geq T C^{f i} .
$$

\section{EXAMPLE 1}

As mentioned earlier, in the first step we assume only stepbids, the parameters of which are as in Table I.

\begin{tabular}{|c|c|c|}
\hline \multicolumn{3}{|c|}{$q_{p}^{f i, j}$} \\
\hline period & 1 & 2 \\
\hline f1-1 & 50 & 40 \\
f1-2 & 35 & 50 \\
\hline
\end{tabular}

\begin{tabular}{|c|c|c|}
\hline \multicolumn{3}{|c|}{$p_{p}^{f i, j}$} \\
\hline period & 1 & 2 \\
\hline f1-1 & 35 & 40 \\
f1-2 & 33 & 42 \\
\hline
\end{tabular}

TABLE I

THE OFFERED AMOUNTS $\left(q_{p}^{f i, j}\right)$ AND PRICES $\left(p_{p}^{f i, j}\right)$ FOR THE TWO BID PROFILES IN EXAMPLE 1.

Let us furthermore suppose that the fix cost component of the generating unit in question is $F^{f 1}=2000$, and the variable costs are $V_{1}^{f 1}=V_{2}^{f 1}=25$.

In this case, assuming various MCPs for the two periods, we may analyze the fulfillment of the MIC condition described in eq. (7). The results are depicted in Fig. 1.

As it can be seen in this figure, the MCP space may be partitioned into regions, according to whether the first, the second, or both profiles meet the MIC criteria.

Theoretically, it is possible that if the MCP is equal to the offer price, the fulfillment of the MIC condition is dependent 


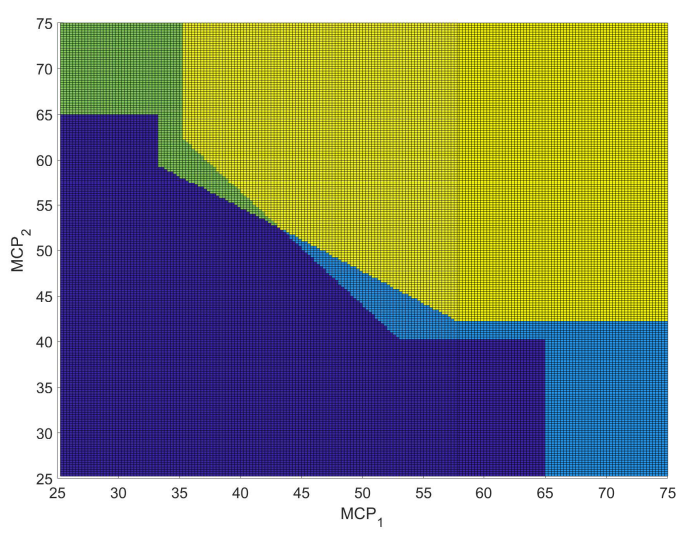

Fig. 1. Fulfilment of MIC conditions in various regions of the MCP space, in the case of example 1 . The dark blue area represents the MCP configurations when none of the bid profiles meets the MIC condition (so both are rejected). In the light blue area profile 1 , while in the green area profile 2 meets the MIC criterion. In the yellow area, both profiles meet the MIC.

on the rate of the (potentially partial) acceptance - however this scenario is not relevant in the case of the proposed example.

In the first two cases, it is straightforward that the first, or the second profile may be accepted respectively. In the third case, when both profiles meet the MIC criteria, the one resulting in the higher social welfare will be selected by the market clearing algorithm.

\section{EXAMPLE 2}

In our second example we consider ramp bids, and show that the proposed concept may be generalized also for this case. Let us note that in this case the inequalities of the income formulation described in eq. (4) and (5) may not be used, thus the resulting model will be no more linear.

We assume that the cost parameter of the unit are the same as before.

Let us suppose the ramp bids depicted in Figs. 2 and 3.

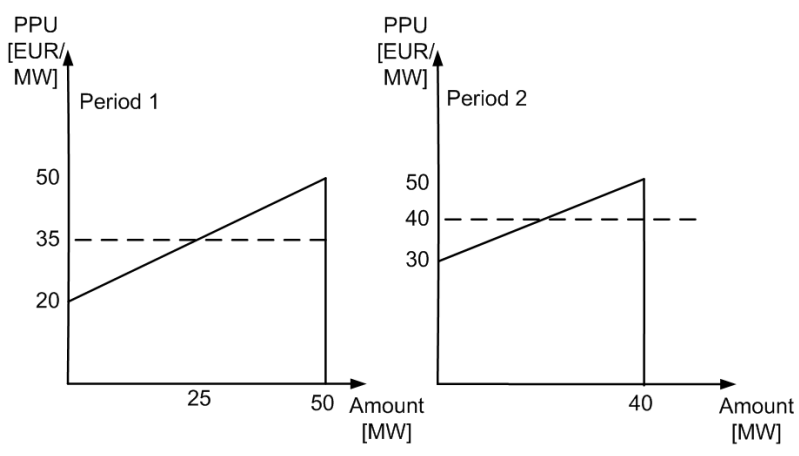

Fig. 2. Ramp bids of bid profile 1 . The original step bids are depicted by dashed lines.

We may consider the same scenarios as in the previous example. Fig. 4 depicts the regions of the MCP space where none, one or both profiles fulfill the MIC condition. We can

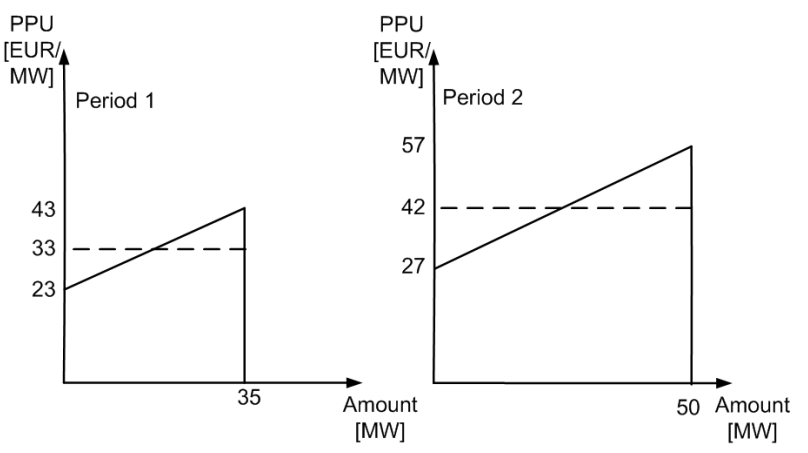

Fig. 3. Ramp bids of bid profile 2. The original step bids are depicted by dashed lines.

see in Fig. 4 that the boundaries between the areas are no more linear.

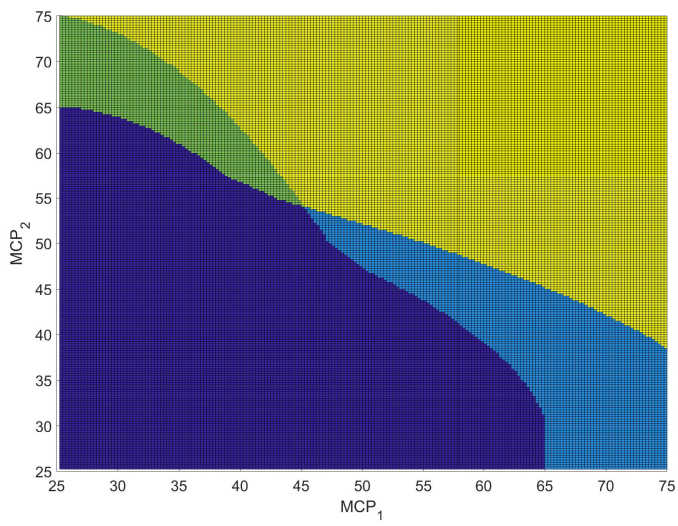

Fig. 4. Fulfilment of MIC conditions in various regions of the MCP space, in the case of example 2 . The dark blue area represents the MCP configurations when none of the bid profiles meets the MIC condition. In the light blue area profile 1, while in the green area profile 2 meets the MIC criterion. In the yellow area, both profiles meet the MIC.

\section{DISCUSSION}

\section{A. Flexibility improvement}

As it can be seen in Figs 1 and 4, the acceptance region of the flexible bid (union of the not dark-blue areas) may be significantly larger compared to either of the single MIC bids (union of the yellow and the green/light blue areas). This shows that in the presence of price-uncertainty, flexible production bids give an increased probability for the generating unit to get one of its bid profiles accepted in the market, thus contribute to an efficient dispatch. As shown in Fig. 4, this flexibility increases with the application of ramp bids, compared to the simple step-bid case.

\section{B. Computational issues}

Regarding the more simple case when only step bids are allowed (Example 1), the proposed bid structure and the implied optimization problem for market clearing may be implemented in a fully linear framework. Flexible production 
bids however increase the number of binary variables. On the one hand, for every profile $j$ in the flexible bid $i$ induces a binary variable $u^{f i, j}$ used for the description of the exclusive nature of the profiles (see eq. (2)), if we have $n_{p}$ profiles for flexible bid this means $n_{p}$ binary variables.

The implementation of the implication constraints in the inequalities of the income (4), (5) also imply $2 n_{p} T$ auxiliary binary variables used in the big-M constraints.

In addition, for the implementation of the minimum income condition (7) also $n_{p}$ auxiliary binary variables are needed.

All together a flexible bid implies $2 n_{p}(T+1)$ binary variables, where $n_{p}$ is the number of bid profiles and $T$ is the number of periods involved. Here for the aim of simplicity we assumed that every profile is defined for the same periods. This however, is not a necessary condition for the application of the proposed framework: profiles may include different periods, and the concept may be used without any modification - of course, the number of indicated binary variables may not be so simply calculated in this case.

In the case of Example 2, the resulting problem will be a Mixed-Integer Quadratic Program, which is the spectrum of EUPHEMIA [3].

\section{CONCLUSiOns}

In this paper we introduced a possible new class of complex bids in day-ahead electricity markets, called flexible order. A flexible order consists of multiple bid profiles, each may be viewed as an MIC bid. The resulting MCPs determine the set of bid profiles which meet the MIC criteria, thus they may be accepted. If multiple bid profiles turn out to be feasible, the market clearing algorithm will choose the one resulting in the highest possible social welfare.

We have shown in the case of simple examples that the acceptance region of the flexible order (interpreted in the MCP space) is potentially larger than the acceptance region of any single MIC bid corresponding to the bid profiles. We argue that under price-uncertainty the proposed bid type may be a useful tool for generating units with start-up cost to account for various MCP outcomes, and increase the flexibility of bidding in the electricity market.

\section{ACKNOWLEDGMENTS}

This work has been supported by the Fund PD 123900 of the Hungarian National Research, Development and Innovation Office, by the János Bolyai Research Scholarship of the Hungarian Academy of Sciences and by the Fund KAP191.1-ITK of the Pázmány Péter Catholic University.

\section{REFERENCES}

[1] D. Finon and F. Roques, "European electricity market reforms: The visible hand of public coordination," Economics of Energy \& Environmental Policy, vol. 2, no. 2, pp. 107-124, 2013.

[2] J.-M. Glachant and F. Lévêque, "Electricity internal market in the european union: What to do next?" Electricity
Reform in Europe. Towards a Single Energy Market, 2009.

[3] "Euphemia public description," Technical report, Tech. Rep., 2015. [Online]. Available: http://www.belpex.be/wp-content/uploads/Euphemiapublic-description-Nov-2013.pdf

[4] L. Meeus, "Power exchange auction trading platform design," Ph.D. dissertation, Katholieke Universiteit, Leuven, 2006.

[5] P. N. Biskas, D. I. Chatzigiannis, and A. G. Bakirtzis, "European electricity market integration with mixed market designs-part i: Formulation," IEEE Transactions on Power Systems, vol. 29, no. 1, pp. 458-465, 2014.

[6] P. N. Biskas, D. I. Chatzigiannis, and A. G. Bakirtzis, "European electricity market integration with mixed market designspart ii: Solution algorithm and case studies," IEEE Transactions on Power Systems, vol. 29, no. 1, pp. 466-475, Jan 2014.

[7] Á. Sleisz and D. Raisz, "Clearing algorithm for minimum income condition orders on european power exchanges," in Power and Electrical Engineering of Riga Technical University (RTUCON), 2014 55th International Scientific Conference on. IEEE, 2014, pp. 242-246.

[8] — , "Efficient formulation of minimum income condition orders on the all-european power exchange," Periodica Polytechnica Electrical Engineering and Computer Science, vol. 59, no. 3, pp. 132-137, 2015.

[9] — , "Complex supply orders with ramping limitations and shadow pricing on the all-european day-ahead electricity market," International Journal of Electrical Power \& Energy Systems, vol. 83, pp. 26-32, 2016.

[10] Á. Sleisz, D. Divényi, and D. Raisz, "New formulation of power plants general complex orders on european electricity markets," Electric Power Systems Research, vol. 169, pp. 229-240, 2019.

[11] P. Cramton, "Electricity market design," Oxford Review of Economic Policy, vol. 33, no. 4, pp. 589-612, 2017.

[12] J. C. Richstein, C. Lorenz, and K. Neuhoff, "An auction story: How simple bids struggle with uncertainty," 2018. 